\title{
Action Observation Training to Improve Activities of Daily Living and Manipulation Skills in Children with Acquired Brain Injury Secondary to an Oncologic Process: A Prospective Case Series Clinical Study
}

\author{
Paula Serrano-GonzÁlez, MSc, OT ${ }^{1,2}$, César Cuesta-García, PhD ${ }^{1,4}$, Eva Girol-LóPez, PT ${ }^{2}$ and \\ Ferran CUENCA-MARTÍNEZ, $\mathrm{PhD}^{3}$ \\ ${ }^{1)}$ Department of Occupational Therapy, Centro Superior de Estudios Universitarios La Salle, Universidad Autónoma de Madrid, \\ Spain \\ ${ }^{2)}$ Niño Jesús University Children's Hospital, Spain \\ ${ }^{3)}$ Centro Superior de Estudios Universitarios La Salle, Universidad Autónoma de Madrid, Spain \\ 4) Occupational Thinks Research Group, Centro Superior de Estudios Universitarios La Salle, Universidad Autónoma de Madrid, \\ Spain
}

\begin{abstract}
Objective: Among solid tumours, medulloblastoma is the most common of the posterior fossa neoplasms, given that it represents $15 \%-20 \%$ of childhood brain tumours. The main aim of the present study was to assess the effects of action observation training on the activities of daily living (ADL) and the manipulation skills of children with acquired brain injury secondary to an oncological process. Methods: We recruited a consecutive convenience sample of 5 patients diagnosed with acquired brain injury secondary to an oncological process. ADL and manipulation skills were assessed using the ABILHAND-Kids and JebsenTaylor Hand Function Test, respectively. After conducting the initial evaluation, we planned the intervention, which lasted 10 weeks and consisted of 40 sessions for each participant, 10 in the occupational therapy department and 30 at home. After completing the intervention, we re-evaluated the main variables. Results: Overall, the results of the postintervention ABILHAND-Kids questionnaire showed a 5-point improvement, with a statistically significant difference and a large effect size. Eighty per cent of the sample showed better results in the total score, with differences between 4 and 8 points. In relation to manual dexterity, as measured by the Jebsen-Taylor Hand Function Test, there were no significant changes, except in one of the participants. Conclusion: It appears that action observation training can elicit positive changes with respect to the development of ADL, but the influence on manual dexterity was almost nonexistent.
\end{abstract}

Key words: Medulloblastoma, Action observation, Activities of daily living, Brain injury

(Phys Ther Res 00: 00-00, 0000)

The Central Brain Tumor Registry of the United States reports that the incidence of medulloblastoma in patients up to 19 years of age is up to 48 for girls and 75 for boys per

Received: July 17, 2021

Accepted: October 22, 2021

Advance Publication by J-STAGE: January 12, 2022

Correspondence to: César Cuesta García, Department of Occupational Therapy, Centro Superior de Estudios Universitarios La Salle, Universidad Autónoma de Madrid, Spain, 28049 Madrid, Spain \# e-mail: cesar.cuesta@lasallecampus.es

doi: 10.1298/ptr.E10134
100,000 patient-years and accounts for $16 \%$ of all paediatric brain tumours, with medulloblastoma accounting for $40 \%$ of all paediatric cerebellar tumours. The peak incidence of medulloblastoma occurs between 3 and 4 years of age and between 8 and 9 years of age ${ }^{1)}$. Each year in Spain, 1500 new cases of cancer are diagnosed in children younger than 15 years, $15 \%-20 \%$ of which are central nervous system tumours. The annual incidence of these tumours is $2-5$ per 100,000 , with a similar distribution for both sexes $^{2)}$.

Among solid tumours, medulloblastoma is the most 
common of the posterior fossa neoplasms, given that it represents $15 \%-20 \%$ of childhood brain tumours. The approach to these patients is multidisciplinary and begins with surgical resection, with the objective to establish a diagnosis and reduce the tumour volume. Subsequently, patients undergo radiotherapy and/or chemotherapy, the aim of which is to eliminate the cancer cells that persist after surgery and prevent a possible recurrence ${ }^{3)}$. After the surgery phase, complications are treated in other departments, such as the paediatric intensive care unit and the rehabilitation unit. Although survival rates for this type of tumour are high, approximately $25 \%$ of survivors have motor and cognitive complications, including ataxia, balance problems, functional motor deficits, emotional lability and speech dysfunction, which typically appear between the first and second day after the surgery. Together, these symptoms comprise posterior fossa syndrome ${ }^{4)}$.

Among the most affected functional motor deficits are manual skills and abilities and the ability to perform activities of daily living (ADL), which are addressed by occupational therapy. Although few studies have described the role of occupational therapy in treating patients with brain tumours, it is important to achieving the maximum independence possible for children in terms of their ADL. Posture, movement and practical activities are compromised in children with acquired brain damage ${ }^{5}$, which, together with sensory difficulties, makes it difficult for them to properly relate to their environment and they will therefore need help performing their ADL. The activities of occupational therapists are therefore essential in facilitating functional recovery, thereby helping children autonomously recover their abilities.

Movement representation techniques, such as action observation (AO) training, are a set of sensorimotor neurotraining tools widely employed in the field of neurorehabilitation ${ }^{6}$. AO training evokes an internal, real-time simulation of what the observer sees ${ }^{7)}$ and can lead to an activation of areas related to the planning, adjustment and automation of voluntary movements in a manner similar to when the movement actually occurs. AO has been previously proposed for arm motor rehabilitation after stroke ${ }^{8)}$ due to the neurophysiological properties of $\mathrm{AO}$ and its rehabilitative potential. Our hypothesis is that AO training can help improve ADL and manipulation skills in children with acquired brain injury secondary to an oncologic process.

The main aim of the present study was therefore to assess the effects of AO training on ADL and the manipulation skills of children with acquired brain injury secondary to an oncologic process.

\section{Materials and Methods}

\section{Study Design}

The present study was a prospective case series clinical study.

\section{Participants}

The study protocol was approved by the ethics committee of the Niño Jesús University Children's Hospital (Madrid, Spain; CI: R-0011/19). We recruited a consecutive convenience sample of 5 patients diagnosed with acquired brain injury secondary to an oncologic process, specifically a brain tumour (medulloblastoma), from the Niño Jesús University Children's Hospital. Prior to enrolment, all patients were explained the study's objectives and implications and agreed to participate by signing an informed consent document. We reviewed case records dated between October 2018 and June 2019.

The inclusion criteria for this prospective case series were as follows: age between 6 and 18 years; brain tumour diagnosis; undergoing partial or complete tumour resection surgery no more than 30 months prior to the completion of this project; undergoing oncologic therapy and standard occupational therapy at the Niño Jesús University Children's Hospital; an acquired brain injury as a consequence of the oncologic process; and a score of 1 or 2 on the Manual Ability Classification System (MACS). The exclusion criteria consisted of the following: level V in the Gross Motor Function Classification System; and the presence of moderate to severe cognitive and neuropsychological disorders according to the Wechsler Intelligence Scale for Children.

\section{Procedures}

The families attended an information session in which the project and steps to be taken in the coming months were explained. After obtaining the informed consent from the parents, we accessed each child's electronic medical records to collect the necessary information about them and their disease process. The data collected were age, gender, diagnosis, surgical interventions, neurological sequelae, use of drugs, arm involvement, dominance, cognitive level and whether they had been and/or were in a radiotherapy and/or chemotherapy programme.

The Data Protection Law (LOPD 3/2018) was always respected, as well as the European regulation (RGPD 2016/ $679)$ and rights. Once the necessary data had been collected, 2 chosen assessments were administered to determine the starting point of the patients' ADL performance, as well as the manipulation skills they presented at the start of the project.

We provided the families with the ABILHAND-Kids (AK) questionnaire. At the end of the questionnaire, the parents had to prioritise the five activities with which they felt their child had the most difficulties; these activities 


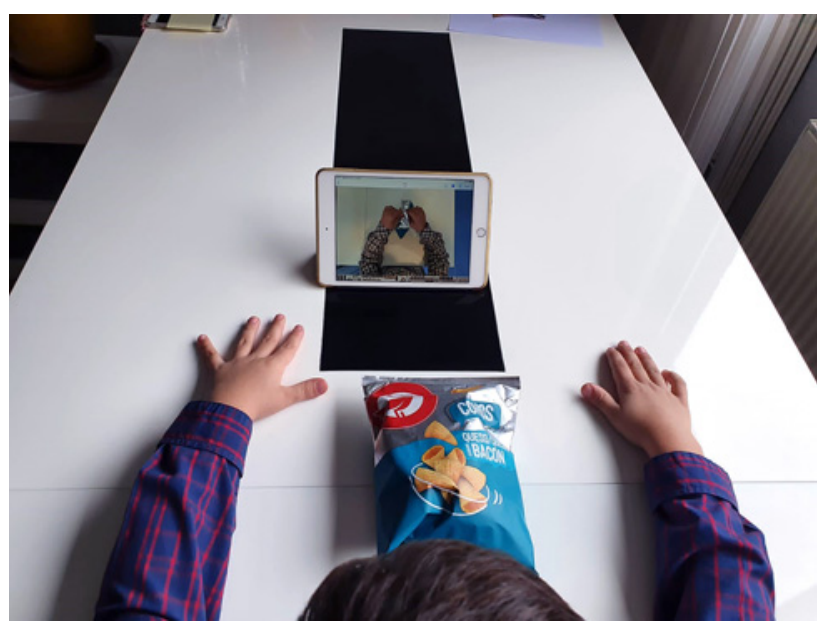

Fig. 1. Intervention representation.

were addressed in the next phase of the project. If there were several activities with similar difficulty, those the parents considered most relevant in their child's environment or those of greatest importance were chosen. The JebsenTaylor Hand Function Test (JTHFT) was then administered.

After conducting the initial assessment, we planned the intervention, which lasted 10 weeks and consisted of 40 sessions for each participant: 10 sessions in the occupational therapy department and 30 at home, where the family conducted the same process performed by the occupational therapist in the department. Each of the 5 activities chosen by the family was performed during 2 consecutive weeks, so that the first activity was addressed during the first and second week, the second activity during the third and fourth week, the third activity during the fifth and sixth week, the fourth activity during the seventh and eighth week, and lastly the fifth activity during the ninth and tenth week. Each session lasted from 20 to $30 \mathrm{~min}$. After finishing the intervention, the main variables were reassessed.

\section{Intervention}

The intervention we employed was AO training. For this purpose, a healthy boy and girl were recorded performing each of the activities in which the participants encountered difficulties. Each patient could choose whether they wanted to work with the boy's video or the girl's video during their intervention, depending on which gender they identified with more. The video was filmed from various perspectives (in third person in both the frontal and lateral plane and in first person). Figure 1 Intervention representation. shows an image of the videos employed in this preliminary study.

The video shows how the healthy boy or girl performs the complete activity and how they perform it by sequencing it in simpler motor acts. Each participant was given only those videos for the activities in which they encountered difficulties and were not given the videos of activities that were not worked on during the intervention. Each motor act was observed for $3 \mathrm{~min}$. Once all the videos of the various motor acts had been watched, the patients had to perform all the activities observed in the videos without interruption.

The AO training was combined with other Physiotherapy and Occupational Therapy interventions. These are summarised in Table 1. The Physiotherapy interventions consisted of balance and postural control training, improved motor conditioning and strength exercises, as well as the vestibular system training. Two of the patients received one Physiotherapy session per week, another patient 2 sessions, another 3 sessions and finally the last one did not receive Physiotherapy. Regarding Occupational Therapy, three of the patients did not receive any intervention and the remaining two patients received one session per week. The Occupational Therapy intervention was based on manual dexterity training and motor control tasks oriented to ADL.

\section{Outcome Measures ABILHAND-Kids}

The AK scale assesses the perception of manual ability of children with cerebral palsy in performing the ADL determined in the scale. In total, the scale contains 21 activities classified into 4 separate levels: question mark (not applicable), impossible, difficult and easy. For the paediatric population, the questionnaire is answered by the parents, who provide their perception of their child's manual ability difficulty when performing the proposed ADL. The AK scale shows excellent internal consistency $(\alpha=0.94)$ and test-retest reliability (Pearson's $r=0.91)^{9)}$. This scale has also shown adequate convergent validity with respect to the MACS test $(r=0.88)^{10)}$. Questionnaires that measure the functional abilities of children through their parents have been shown to be important in the evaluation of clinical changes. This is because children present a more dichotomous perception of their own abilities, allowing the measurement of ability but not of daily performance ${ }^{11,12)}$.

In the present study, the 21 items of the scale were not evaluated. Instead, parents were asked to indicate, among the 21 activities, the five that presented the greatest difficulties for the children, which were the therapeutic target of the subsequent intervention. Therefore, the results are shown in terms of time required to perform the activity, rather than the degree of performance. These activities could coincide among different children and, were the target of the subsequent intervention.

\section{Jebsen-Taylor Hand Function Test}

The JTHFT test measures the time it takes the child to perform each task with both the affected and unaffected arm. The objective of the JTFHT is to assess uni-manual hand functions necessary for activities of daily living. The test consists of 7 subtests, performed with both the non- 


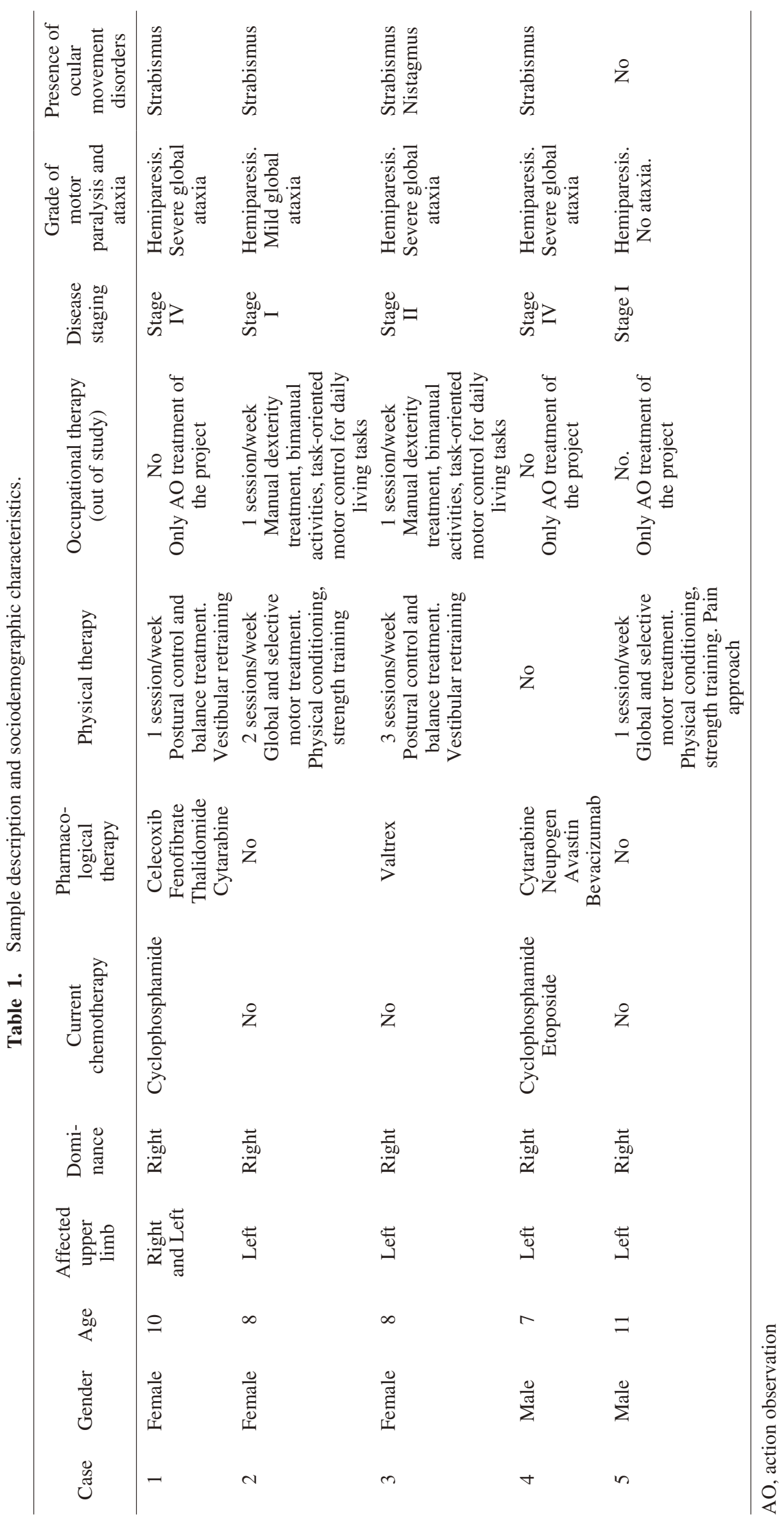


dominant and dominant hand: writing, turning letters, picking up small objects and placing them in a container, stacking chips, stimulated feeding, and moving light and heavy objects ${ }^{13)}$. In this study, all items were tested except writing. There is evidence that the JTHFT is a valid assessment tool for children aged 6 to 18 years with brain injury ${ }^{14)}$. The JTHFT is reliable in a brief assessment period in children with brain injury and is responsive to changes in a brief period of intensive therapy. The minimal clinically important difference (MCID) is $54.7 \mathrm{~s}$ in the affected limb ${ }^{15}$.

\section{Data Analysis}

We analysed data from the 5 patients using IBM SPSS Statistics ver. 25.0 (IBM Co., Armonk, NY, USA). We employed descriptive statistics to summarise the data of the continuous variables, which are presented as mean \pm standard deviation and a 95\% confidence interval. We employed Student's t-test to compare the outcomes of the continuous variables, calculating the effect sizes (Cohen $d$ ) for the outcome variables. According to Cohen's method, the magnitude of the effect was classified as small (0.20-0.49), medium (0.50-0.79), or large $(\geq 0.8)$. A value of $p<0.05$ was considered statistically significant ${ }^{16)}$.

\section{Results}

Our sample was composed of 5 patients ( 3 girls and 2 boys), with a mean age of 8.8 years (range 7-11 years) and a common medical diagnosis of medulloblastoma. After surgery, the patients presented hemiparesis, with predominant involvement of the left arm. The entire sample underwent radiotherapy and chemotherapy. Currently, none of the patients are undergoing radiotherapy, although $40 \%$ are still undergoing chemotherapy. In terms of other drugs, $60 \%$ of the sample continues with pharmacological therapy (Table 1), and none of the participants were treated with botulinum toxin. In individual terms, Table 2 shows the preintervention and postintervention data.

\section{Case 1}

For this patient, the 5 most difficult ADL were buttoning up a shirt, opening a bag of potato crisps, unwrapping a chocolate bar, opening a jam jar and unscrewing a bottle cap. After the treatment, the times for all of the activities were reduced. The most significant time differences were the activities of buttoning up a shirt and unscrewing a bottle cap, which took 56 and $98.2 \mathrm{~s}$ less, respectively. Moreover, after administering the AK post-treatment scale, we observed a score increase of 5 points. In terms of the JTHFT results, there was an increase in time with respect to the preintervention results, indicating no improvement in manipulation dexterity.

\section{Case 2}

The 5 ADL that the patient had the most difficulty with were buttoning up a shirt, opening a bag of potato crisps, buttoning up the trouser buttons, buttoning up a jacket and unscrewing a bottle cap. The participant managed to reduce the times for all the trained activities during the intervention, with a marked reduction in time spent buttoning up a shirt (37 s) and unscrewing a bottle cap (128.1 $\mathrm{s})$. In the postintervention assessment, the increase in $\mathrm{AK}$ score indicated that the performance of the proposed activities had improved (by 4 points). As for the JTHFT results, there was no demonstrated improvement in manual dexterity.

\section{Case 3}

The 5 ADL that the patient had the most difficulty with were buttoning up the trouser buttons, sharpening a pencil, putting toothpaste on a toothbrush, filling a glass of water, and unscrewing a bottle cap. The time was also reduced for all activities in which the intervention was performed. There was a marked reduction in the time to button up trouser buttons (25.7 s) and unscrew a bottle cap (48.7 s). The AK results also confirmed that the performance of these activities had improved by 8 points, a result that was the highest in the entire case series. As in the previous cases, the JTHFT results showed no change in manipulation dexterity.

\section{Case 4}

The 5 most difficult ADLs for this patient were buttoning up a shirt, opening a bag of potato crisps, buttoning up the trouser buttons, sharpening a pencil and filling a glass of water. In this case, there was a considerable decrease in the time spent on 3 of the 5 activities addressed during the intervention: buttoning up the shirt, buttoning up the trouser buttons and opening a bag of potatoes (improvement of approximately 8, 6 and $1 \mathrm{~min}$, respectively). In the post-intervention assessment on the AK scale, there was a 7-point increase, which confirms that the performance of the addressed activities after the intervention had improved. In terms of the JTHFT results, this is the only participant who showed reduced time spent on the test (approximately $1 \mathrm{~min}$ ) compared with the initial assessment.

\section{Case 5}

The 5 ADLs where the patient presented the most difficulties were buttoning up a shirt, buttoning up the trousers, opening a toothpaste dispenser, sharpening a pencil, and opening a bread bag. The time spent on these tasks improved but to a lesser extent than the other cases. The activities in which the patient managed to reduce the time spent the most were buttoning up a shirt and opening a bread bag (19.5 and $10.9 \mathrm{~s}$, respectively). In terms of the AK results, there were no differences compared with the re- 


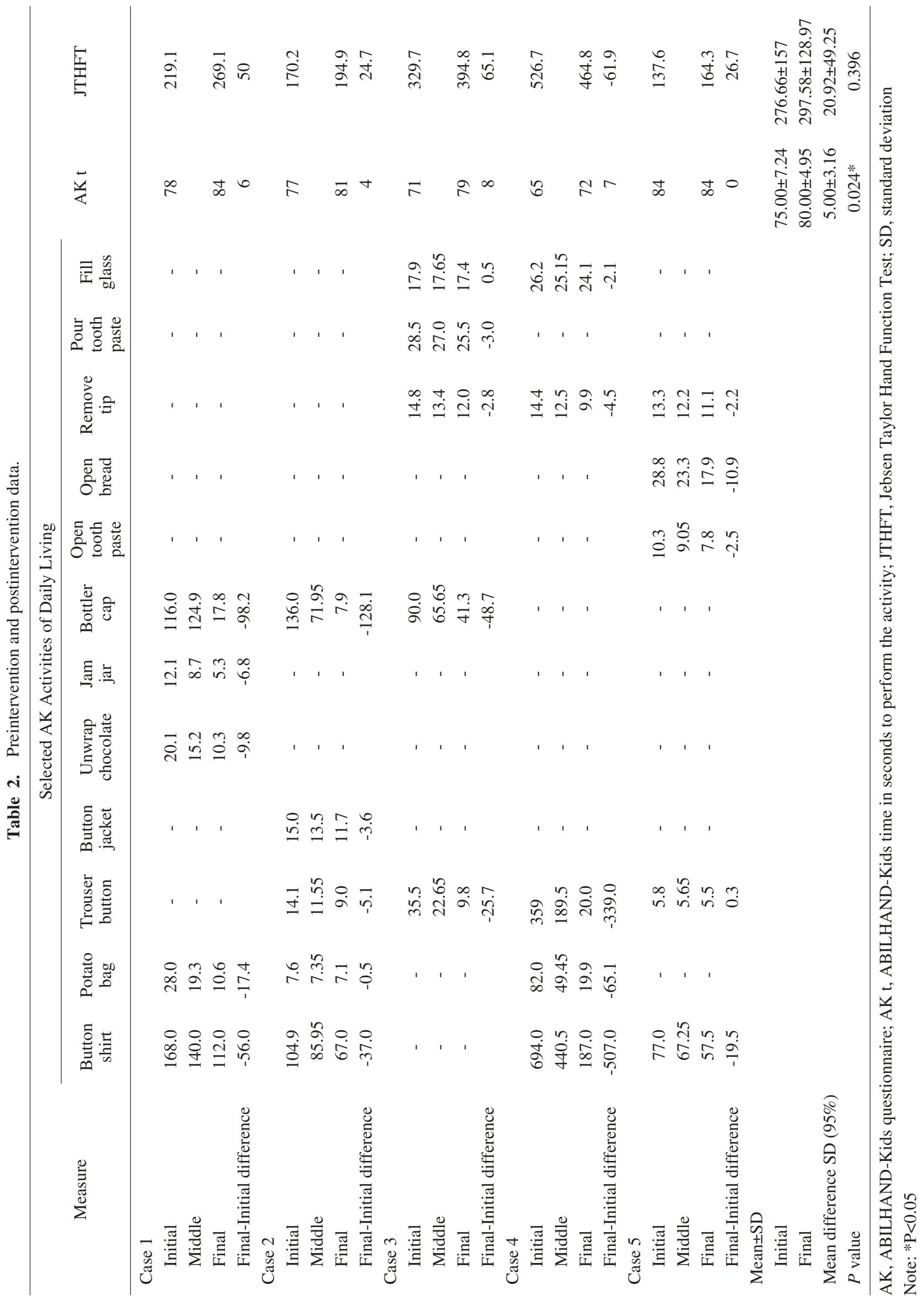


sults of the preintervention assessment. As to the times obtained in the JTHFT, there was an increase compared with the pre-intervention assessment. Regarding adherence, all patients completed all OA training sessions. Adherence was complete in all cases.

\section{Statistical Analysis}

Regarding the AK variable, statistically significant pre- and post-intervention differences were found with a large effect size ( $\mathrm{p}=0.024 d=-0.80 ; \mathrm{t}=2.04$ ) (Table 2). However, with regard the JTHFT variable, no statistically significant differences were found $(\mathrm{p}=0.396)$ (Table 2).

\section{Discussion}

The main aim of the present study was to assess the effects of AO training in ADL and manipulation skills in children with acquired brain injury secondary to an oncologic process. Overall, the results of the postintervention AK questionnaire showed an improvement of 5 points, with a statistically significant difference and large effect size. Eighty per cent of the sample showed better results in the total score, with differences between 4 and 8 points. The remaining $20 \%$ ( 1 child) obtained identical results for the preintervention and postintervention assessments. After the intervention, all the sub-tasks trained with the AO approach showed better results in terms of execution time. In relation to manual dexterity as measured by the JTHFT, there were no significant changes except in one of the participants.

All 5 participants were administered a therapeutic intervention of $20 \mathrm{~h}$, distributed over 10 weeks. All participants demonstrated significant changes in trained ADL through the AO technique as measured by the AK. The study by Bleyenheuft et al. of a cohort of 80 children with unilateral cerebral palsy undergoing intensive interventions showed (through the AK questionnaire) improvements in all participants, which was greater in magnitude in the younger children (6-12 years) and with MACS levels above $\mathrm{II}^{17)}$. Although the clinical diagnoses of the 2 studies differed, our participants had MACS levels between I and II, as well as ages between 7 and 11 years. These changes could be explained by the greater neuroplastic capacity at earlier stages of progression and at younger ages.

In addition, our results only showed significant changes in manual dexterity in 1 patient, with no correlations with ADL performance as measured by AK and manual dexterity. The study conducted by Bleyenheuft et al. showed similar results ${ }^{17)}$. It is important to stress that the MCID for the JTHFT in children aged 13-19 years is 54.7. Given that our sample's mean age was 8.8 years (7-11 years) we cannot dispute these data. Regardless, the variations in our study participants do not reach these differences in their pretreatment and post-treatment differences, except for one of the participants, whose results exceeded the MCID.

Another important aspect in our intervention is the focus on goals chosen by the children, increasing their motivation with task-oriented training and participation. For Novak et al., task-oriented, goal-directed training and home programs are highly recommended interventions for children with neuromotor impairment due to cerebral palsy $^{18)}$.

AO training is a sensorimotor neurotraining tool focused on provoking neurophysiological changes at the cortical level, that is, favouring a process of neuroplasticity with respect to the processes related to the planning, adjustment and automation of voluntary movements ${ }^{7}$. In this population, it is important to be able to implement these techniques, known as movement representation methods, to elicit greater neurophysiological activity at the cerebral level. A recently published umbrella review with the statistical aggregation of meta-analytic results showed that movement representation methods such as AO training and motor imagery could elicit an improvement in ADL in a population with neurological conditions ${ }^{19)}$, which agrees with the findings in this case series study. ADL, as with any task that is amenable to a learning process, can derive benefits from AO training. For example, Cuenca-Martínez et al. found that $\mathrm{AO}$ training alone is an effective method for learning manual gestures for at least 4 months after completion of the intervention ${ }^{20)}$. There is ample evidence to support the use of movement representation methods to elicit a specific motor learning process ${ }^{21-23)}$.

The recent study conducted by Buccino et al. showed the efficacy of a 3-week intervention with AO for the rehabilitation of arm motor functions involved in $\mathrm{ADL}^{24)}$. The sample included children with cerebral palsy aged 5 to 11 years, who when treated showed increased neurophysiological activation of a parietal-premotor circuit for handobject interactions. In addition, changes were found through neuroimaging in the left and right premotor cortex, left supramarginal gyrus and left superior temporal. Our therapeutic intervention had to be adapted to the contextual conditions and to be able to intervene in a larger number of ADL.

\section{Limitations}

This study has several important limitations that need to be considered. First, this is a case series, and all results should be taken with caution due to the small sample size. It is likely that the results could vary significantly with a larger sample. Second, due to the type of disease, the children underwent other medical interventions that might have influenced the results. We collected only the information that was reported, but this should be considered as highly important confounding variables. In addition, 1 child underwent occupational therapy outside the study, which might also influence the results, making them difficult to attribute 
to the intervention alone. Finally, basic, and instrumental skills were not assessed, only manual dexterity. Future studies should assess the overall ADL for each patient.

\section{Conclusions}

Based on the results, it appears that AO training could elicit positive changes with respect to the development of ADL, but there was almost no influence on manual dexterity in the patients diagnosed with acquired brain injury secondary to an oncologic process. Although these conclusions are valid for a series of 5 cases, the results should be taken with great caution.

Acknowledgments: The authors would like to thank Centro Superior de Estudios Universitarios CSEU La Salle for its services in editing this manuscript.

Funding: No sources of funding were used to assist in the preparation of this article.

Conflict of Interest: The authors declare that they have no conflicts of interest. This research study did not receive any specific grant from funding agencies in the public, commercial, or not-for-profit sectors.

\section{References}

1) Crawford JR, MacDonald TJ, et al.: Medulloblastoma in childhood: new biological advances. Lancet Neurol. 2007; 6: 10731085.

2) Villarejo F and Martínez-Lage J: Tumores cerebrales en niños. Pediatría Integr. 2012; 16: 475-486.

3) Douglas-Akinwande AC, Payner TD, et al: : Medulloblastoma mimicking Lhermitte-Duclos disease on MRI and CT. Clin Neurol Neurosurg. 2009; 111: 536-539.

4) Robertson PL, Muraszko KM, et al.: Incidence and severity of postoperative cerebellar mutism syndrome in children with medulloblastoma: A prospective study by the Children's Oncology Group. J Neurosurg. 2006; 105: 444-451.

5) Kushner DS and Amidei C: Rehabilitation of motor dysfunction in primary brain tumor patients. Neuro-Oncology Pract. 2015; 2: 185-191.

6) Zhang B, Kan L, et al.: The effects of action observation training on improving upper limb motor functions in people with stroke: A systematic review and meta-analysis. PLoS One. 2019; 14: e0221166.

7) Buccino G: Action observation treatment: a novel tool in neurorehabilitation. Philos Trans R Soc B Biol Sci. 2014; 369: 20130185.

8) Small SL, Buccino G, et al.: Brain repair after stroke - A novel neurological model. Nat Rev Neurol. 2013; 9: 698-707.

9) Arnould C, Penta M, et al.: ABILHAND-Kids: A measure of manual ability in children with cerebral palsy. Neurology. 2004;
63: 1045-1052.

10) Öhrvall AM, Krumlinde-Sundholm L, et al.: Exploration of the relationship between the Manual Ability Classification System and hand-function measures of capacity and performance. Disabil Rehabil. 2013; 35: 913-918.

11) M W and K S: Upper limb function in everyday life of children with cerebral palsy: description and review of parent report measures. Disabil Rehabil [Internet]. 2015 [cited 2021 Sep 21]; 37:1353-1361. Available from: https://pubmed.ncbi.nlm.nih.go v/25264734/

12) Arnould C, Penta M, et al.: Hand impairments and their relationship with manual ability in children with cerebral palsy. J Rehabil Med. 2007; 39: 708-714.

13) Taylor N and Sand PL JR: Evaluation of hand function in children. Am Congr Rehabil Med. 1973; 54: 129-135.

14) Tofani M, Castelli E, et al.: Examining Reliability and Validity of the Jebsen-Taylor Hand Function Test Among Children With Cerebral Palsy. Percept Mot Skills. 2020; 127: 684-697.

15) Araneda R, Ebner-Karestinos D, et al.: Reliability and responsiveness of the Jebsen - Taylor Test of Hand Function and the Box and Block Test for children with cerebral palsy. Dev Med Child Neurol. 2019; 61: 1182-1188.

16) Cohen J: LEA (ed): Statistical power analysis for the behavioral sciences. Second, Hillsdale NJ, 1988, pp. 82.

17) Bleyenheuft Y, Ebner-Karestinos D, et al.: Intensive upper- and lower-extremity training for children with bilateral cerebral palsy: a quasi-randomized trial. Dev Med Child Neurol. 2017; 59: 625-633.

18) Novak I, Morgan C, et al: : State of the Evidence Traffic Lights 2019: Systematic Review of Interventions for Preventing and Treating Children with Cerebral Palsy. Vol. 20, Current Neurology and Neuroscience Reports, Springer, 2020, pp. 1-21.

19) Herranz-Gómez A, Gaudiosi C, et al.: Effectiveness of motor imagery and action observation on functional variables: An umbrella and mapping review with meta-meta-analysis. Neurosci Biobehav Rev. 2020; 118: 828-845.

20) Cuenca-Martínez F, Suso-Martí L, et al.: Effects of movement representation techniques on motor learning of thumbopposition tasks. Sci Rep. 2020; 10: 12267.

21) La Touche R, Sánchez-Vázquez M, et al.: Instruction Modes for Motor Control Skills Acquisition: A Randomized Controlled Trial. J Mot Behav. 2019; 1-12.

22) Cuenca-Martínez F, La Touche R, et al.: Mental practice in isolation improves cervical joint position sense in patients with chronic neck pain: a randomized single-blind placebo trial. PeerJ. 2019; 7: e7681.

23) Lagravinese G, Bisio A, et al.: Learning by observing: the effect of multiple sessions of action-observation training on the spontaneous movement tempo and motor resonance. Neuropsychologia. 2017; 96: 89-95.

24) Buccino G, Molinaro A, et al: : Action Observation Treatment Improves Upper Limb Motor Functions in Children with Cerebral Palsy: A Combined Clinical and Brain Imaging Study. Neural Plast. 2018; 4843985. 